\title{
DOSim: An R package for similarity between diseases based on Disease Ontology
}

\author{
Jiang Li ${ }^{\dagger}$, Binsheng Gong ${ }^{\dagger}$, Xi Chen, Tao Liu, Chao Wu, Fan Zhang, Chunquan Li, Xiang Li, Shaoqi Rao and Xia Li
}

\begin{abstract}
Background: The construction of the Disease Ontology (DO) has helped promote the investigation of diseases and disease risk factors. DO enables researchers to analyse disease similarity by adopting semantic similarity measures, and has expanded our understanding of the relationships between different diseases and to classify them. Simultaneously, similarities between genes can also be analysed by their associations with similar diseases. As a result, disease heterogeneity is better understood and insights into the molecular pathogenesis of similar diseases have been gained. However, bioinformatics tools that provide easy and straight forward ways to use DO to study disease and gene similarity simultaneously are required.

Results: We have developed an R-based software package (DOSim) to compute the similarity between diseases and to measure the similarity between human genes in terms of diseases. DOSim incorporates a DO-based enrichment analysis function that can be used to explore the disease feature of an independent gene set. A multilayered enrichment analysis ( $G O$ and KEGG annotation) annotation function that helps users explore the biological meaning implied in a newly detected gene module is also part of the DOSim package. We used the disease similarity application to demonstrate the relationship between 128 different DO cancer terms. The hierarchical clustering of these 128 different cancers showed modular characteristics. In another case study, we used the gene similarity application on 361 obesity-related genes. The results revealed the complex pathogenesis of obesity. In addition, the gene module detection and gene module multilayered annotation functions in DOSim when applied on these 361 obesity-related genes helped extend our understanding of the complex pathogenesis of obesity risk phenotypes and the heterogeneity of obesity-related diseases.

Conclusions: DOSim can be used to detect disease-driven gene modules, and to annotate the modules for functions and pathways. The DOSim package can also be used to visualise DO structure. DOSim can reflect the modular characteristic of disease related genes and promote our understanding of the complex pathogenesis of diseases. DOSim is available on the Comprehensive R Archive Network (CRAN) or http://bioinfo.hrbmu.edu.cn/ dosim.
\end{abstract}

\section{Background}

The past several decades have seen a number of methods applied to the computation of similarities between diseases [1-4]. The early work used clinical phenotypes or diagnosed information. For example, Kalaria [1] ascertained similarities between Alzheimer's disease and vascular dementia by studying the similarities between disease symptoms and pathological result. More recently, with the availability of large-scale knowledge

\footnotetext{
*Correspondence: shaoqirao@yahoo.com; lixia@hrbmu.edu.cn

† Contributed equally

College of Bioinformatics Science and Technology, Harbin Medical University, 194 Xuefu Road, Harbin 150081, China
}

bases such as the Online Mendelian Inheritance in Man (OMIM) [5] and the Genetic Association Database (GAD) [6], scientists are able to explore the genetic similarity between diseases. In 2009, Liu et al. [7] revealed similarities between diseases by combining both genetic (data from GAD [6]) and environmental (data from Medical Subject Headings, MeSH [8]) factors and, by mining for disease etiologies, created a new concept named the "etiome". Zhang and his colleagues [9] used a text-based method to build up a human disease phenotype network in which a disease was represented by a feature vector and the similarities between two diseases were calculated as the cosine of the angle between their
C Biomed Central

() 2011 Li et al; licensee BioMed Central Ltd. This is an Open Access article distributed under the terms of the Creative Commons Attribution License (http://creativecommons.org/licenses/by/2.0), which permits unrestricted use, distribution, and reproduction in any medium, provided the original work is properly cited. 
corresponding feature vectors. However, little work has been done to apply semantic similarity measures between diseases using ontology, another way to analyze relationship between diseases.

Understanding similarities between genes has a significant role to play in disease research. One hypothesis states that genes associated with similar diseases have similar functions; the greater the gene similarity the higher the probability that the genes are associated with similar similarity. However, current methods to determine gene similarity rely on sequence similarity, gene expression profiles, Gene Ontology (GO) [10] annotations or PubMed abstracts, all of which are derived from normal or partially abnormal conditions and it secludes gene similarity from disease similarity. Thus, a process to determine the similarities between genes in terms of diseases and to map gene similarities to disease similarities would help us better understand the mechanism of complex diseases.

The Disease Ontology (DO) aims to provide an open source ontology for the integration of biomedical data that is associated with human disease [11]. The terms in DO are disease names or disease-related concepts and are organised in a directed acyclic graph (DAG) (Figure 1). Two linked diseases in DO are in an 'is-a' relationship, which means one disease is a subtype of the other linked disease. And the lower a disease is in the DO hierarchy, the more specific the disease term is. A recent work by Osborne and his colleagues [12] in

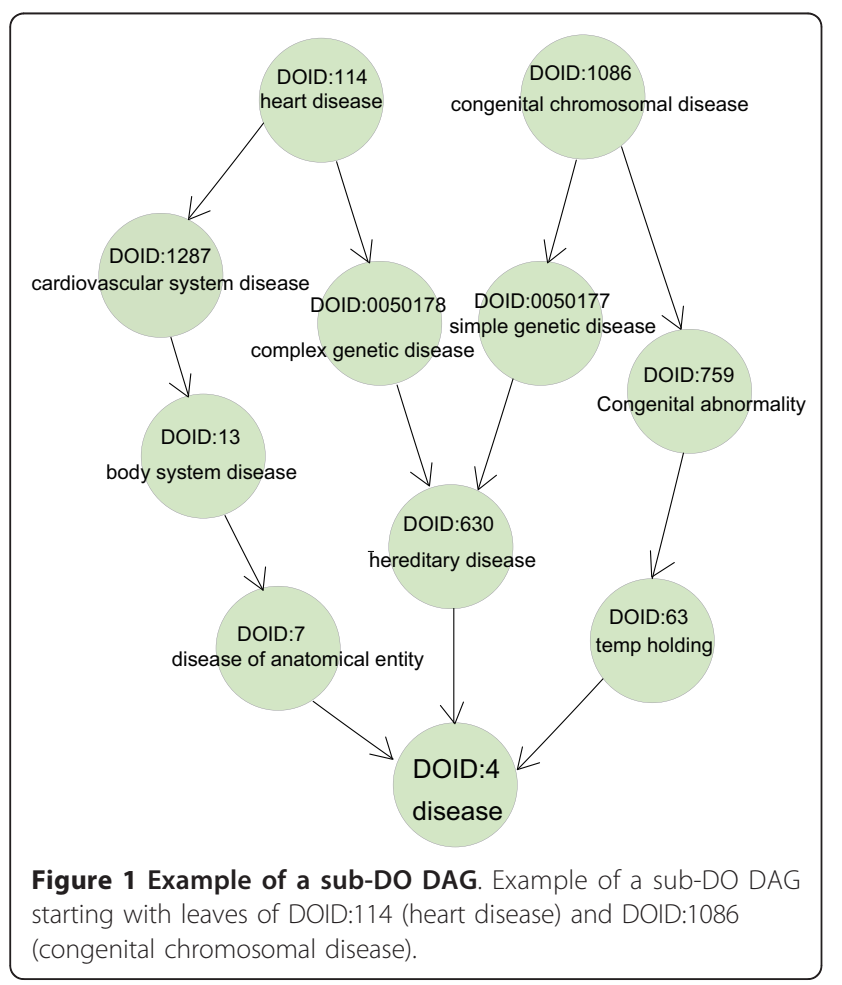

which they used DO to annotate the human genome, further advanced the application of DO. Recently, a simplified vocabulary list, Disease Ontology Lite (DOLite), was shown to give more interpretable results than DO in gene-disease association tests. DOLite has been used in FunDO (Functional Disease Ontology) [13], one of the few bioinformatics tools based on DO that aims to explore disease information implied in the gene set. This work makes it possible to study disease similarity and gene similarity simultaneously in DO using the annotated human genome. Thus, we developed DOSim, an R package for the computation of DObased similarity between diseases in an ontology sense. DOSim was developed on DO, subversion 926; the DO term annotations of the human genes in DOSim were taken from the study of Osborne et al. [12]. A total of 4054 genes have been assigned DO term annotations. Compared with FunDO, DOSim divides functions into three categories: (i) measuring the similarity between diseases (DO terms), (ii) measuring the similarity between human genes in terms of diseases, (iii) other utilities for conducting DO enrichment analysis (similar to FunDO), detecting and annotating DO-directed gene modules, and describing and visualizing DO structures and terms.

\section{Implementation}

\section{Measuring the similarity between diseases}

Terms in DO include disease names and disease-related concepts. Exploring the similarity between them can help us to understand the relatedness between diseases. The past few years have seen an increase in the number of different measures used for the calculation of semantic similarity. Based on the semantic similarity measures in the application of biomedical ontologies reviewed by Pesquita etc al. [14], for general applicability, in DOSim we implemented ten representative semantic similarity measures, which are Resnik measure [15], Lin measure [16], Jiang and Conrath measure (JC) [17], Relevance measure (Rel) [18], Graph Information Content measure (GIC) [19], Information Coefficient similarity measure (simIC) [20], Wang measure [21], modified Resnik measure (CoutoResnik) [22], modified Lin measure (CoutoLin) [22], and modified Jiang and Conrath measure (CoutoJC) [22]. Except for the Wang measure that uses a hybrid measure, the other nine measures are based on information content (IC).

The IC of a term/disease $t$ in the DO database gives a measure of how specific and informative a term/disease is, and is defined as $I C(t)=-\log p(t)$, where $p(t)$ is the number of genes annotated to the term $t$ and its descendants divided by the total number of genes annotated to DO. When characterizing the shared IC between two terms, two concepts, most informative common 
ancestor (MICA) and disjunctive common ancestor (DCA), are widely used[22]. The MICA of two terms $t_{1}$ and $t_{2}$ is the one that possesses the maximum IC among all the common ancestor terms of the two terms. And the DCAs of two terms $t_{1}$ and $t_{2}$ are the MICA of disjunctive ancestors of the two terms, which can be defined as follows:

$$
\begin{aligned}
& \text { DisjCommonAnc }\left(t_{1}, t_{2}\right)=\left\{a_{1} \mid\right. \\
& a_{1} \in \operatorname{CommonAnc}\left(t_{1}, t_{2}\right) \wedge \\
& \forall a_{2}:\left[\left(a_{2} \in \operatorname{CommonAnc}\left(t_{1}, t_{2}\right)\right) \wedge\left(I C\left(a_{1}\right) \leq I C\left(a_{2}\right)\right)\right] \Rightarrow \\
& \left.\left[\left(a_{1}, a_{2}\right) \in\left(\operatorname{DisjAnc}\left(t_{1}\right) \cup \operatorname{DisjAnc}\left(t_{2}\right)\right)\right]\right\}
\end{aligned}
$$

where disjunctive ancestors of the term $t, \operatorname{DisjAnc}(t)$, can be described as that two ancestors $a_{1}$ and $a_{2}$ are disjunctive ancestors of the term $t$ if there is a path from $a_{1}$ to $t$ not passing through $a_{2}$ and a path from $a_{2}$ to $t$ not passing through $a_{1}$. It can be formulated as follows:

$$
\begin{aligned}
& \operatorname{DisjAnc}(t)=\left\{\left(a_{1}, a_{2}\right) \mid\right. \\
&\left(\exists p:\left(p \in \operatorname{Paths}\left(a_{1}, t\right)\right) \wedge\left(a_{2} \notin p\right)\right) \wedge \\
&\left.\left(\exists p:\left(p \in \operatorname{Paths}\left(a_{2}, t\right)\right) \wedge\left(a_{1} \notin p\right)\right)\right\}
\end{aligned}
$$

Then, the shared information of two terms $t_{1}$ and $t_{2}$, Share $\left(t_{1}, t_{2}\right)$, is defined as the average of the IC of the DCAs, formulated as:

$$
\operatorname{Share}\left(t_{1}, t_{2}\right)=\overline{\left\{I C(a) \mid a \in \operatorname{DisjCommonAnc}\left(t_{1}, t_{2}\right)\right\}}
$$

Let $t_{M I C A}$ represent the MICA term of two terms $t_{1}$ and $t_{2}$, then the nine IC-based similarity measures are calculated as follows:

$$
\begin{aligned}
& \operatorname{Sim}_{\operatorname{Re} \text { snik }}\left(t_{1}, t_{2}\right)=I C\left(t_{M I C A}\right) \\
& \operatorname{Sim}_{L i n}\left(t_{1}, t_{2}\right)=\frac{2 \times I C\left(t_{M I C A}\right)}{I C\left(t_{1}\right)+I C\left(t_{2}\right)} \\
& \operatorname{Sim}_{J C}\left(t_{1}, t_{2}\right)=1-\min \left(1, I C\left(t_{1}\right)+I C\left(t_{2}\right)-2 \times I C\left(t_{M I C A}\right)\right) \\
& \operatorname{Sim}_{\operatorname{Re} l}\left(t_{1}, t_{2}\right)=\operatorname{Sim}_{\text {Lin }}\left(t_{1}, t_{2}\right) \times\left(1-p\left(t_{\text {MICA }}\right)\right) \\
& \operatorname{Sim}_{\text {GIC }}\left(t_{1}, t_{2}\right)=\frac{\sum_{t \in\left(\text { Ancestor }\left(t_{1}\right) \cap \text { Ancestor }\left(t_{2}\right)\right)} I C(t)}{\sum_{t \in\left(\text { Ancestor }\left(t_{1}\right) \cup \text { Ancestor }\left(t_{2}\right)\right)} I C(t)} \\
& \operatorname{Sim}_{\text {simIC }}\left(t_{1}, t_{2}\right)=\operatorname{Sim}_{\text {Lin }}\left(t_{1}, t_{2}\right) \times\left(1-\frac{1}{1+\operatorname{IC}\left(t_{\text {MICA }}\right)}\right) \\
& \operatorname{Sim}_{\text {Couto Re snik }}\left(t_{1}, t_{2}\right)=\operatorname{Share}\left(t_{1}, t_{2}\right) \\
& \operatorname{Sim}_{\text {CoutoLin }}\left(t_{1}, t_{2}\right)=\frac{2 \times \operatorname{Share}\left(t_{1}, t_{2}\right)}{\operatorname{IC}\left(t_{1}\right)+\operatorname{IC}\left(t_{2}\right)}
\end{aligned}
$$

$$
\operatorname{Sim}_{\text {CoutolC }}\left(t_{1}, t_{2}\right)=1-\min \left(1, \operatorname{IC}\left(t_{1}\right)+\operatorname{IC}\left(t_{2}\right)-2 \times \operatorname{Share}\left(t_{1}, t_{2}\right)\right)
$$

In the Wang measure, each edge is given a weight according to the types of relationships. For a term $A$, a sub-DAG comprised of the term $A$ and all its ancestor terms can be represented as $D A G_{A}=\left(A, T_{A}, E_{A}\right)$, where $T_{A}$ is the ancestor term set of term $A$ (including $A$ itself) and $E_{A}$ is the set of edges connecting to the terms in $D A G_{A}$. For any term $t$ in $D A G_{A}$, Wang et al. [21] defined the semantic contribution of $t$ to $A, D A(t)$, as the product of all the edge weights in the "best" path from term $t$ to $A$, where the "best" path is the one that maximises the product (the semantic contribution of the term $A$ to itself is set to 1$)$. It can be represented as follow:

$$
\left\{\begin{array}{l}
S_{A}(A)=1 \\
S_{A}(t)=\max \left\{w_{e} \times S_{A}\left(t^{\prime}\right) \mid t^{\prime} \in \operatorname{childrenof}(t)\right\} \text { if } t \neq A
\end{array}\right.
$$

where $w_{e}$ is the semantic contribution factor of edge $e$ ( $e \in E_{A}$ ). It is set between 0 and 1 according to the types of relationships, e.g., "is-a" or "part-of". In DO, there is only one type of relationship, defined as "is-a". In DOSim, we set $w_{e}$ to 0.7 .

The semantic similarity between two terms $A$ and $B$ is then calculated as follows:

$$
\operatorname{Sim}_{\text {Wang }}(A, B)=\frac{\sum_{t \in T_{A} \cap T_{B}}\left(S_{A}(t)+S_{B}(t)\right)}{S V(A)+S V(B)}
$$

where $S V(A)$ (or $S V(B)$ ) is the total semantic contribution of the term $A$ (or $B$ ) in $D A G_{A}$ (or $D A G_{B}$ ), which is calculated as:

$$
\begin{aligned}
& S V(A)=\sum_{t \in T_{A}} S_{A}(t) \\
& S V(B)=\sum_{t \in T_{B}} S_{B}(t)
\end{aligned}
$$

\section{Measuring the similarity between human genes in terms of diseases}

In the DOSim package, the similarity between two genes based on the similarity of their DO term annotation groups is calculated. Each gene is represented by its set of direct DO term annotations, and semantic similarity is calculated between terms in one set and terms in the other (using one of the measures described above). Some methods consider every pairwise combination of terms for the two sets, while others consider only the best-matching pair for each term. Five different methods are implemented in DOSim; they are the arithmetic maxima and average of pairwise similarity between two groups of DO terms describing the 
two genes (Max, Mean) [23], the arithmetic maxima and average between similarities for two directional comparisons of the similarity matrix $S$ of two genes (funSimMax, funSimAvg) [18], and the best-match average approach (BMA) [21] which considers the contributions from the semantically similar terms that annotated the two genes respectively (Formula 23 ).

Let $D O_{1}$ and $D O_{2}$ be the groups of annotation terms for two genes $g_{1}$ and $g_{2}$, and $m$ and $n$ are the number of terms in $D O_{1}$ and $D O_{2}$ respectively. A similarity matrix $S=\left[s_{i j}\right]_{m \times n}$ contains all pairwise similarity scores of mappings from $D O_{1}$ to $D O_{2}$ when you refer to each row and vice verse when you refer to each column. 'rowScore' and 'columnScore' of $S$ are the averages over the row maxima and the column maxima, which give similarity scores for the comparison of $\mathrm{DO}_{1}$ to $\mathrm{DO}_{2}$ and the comparison of $\mathrm{DO}_{2}$ to $D \mathrm{O}_{1}$, respectively.

$$
\begin{aligned}
& \text { rowScore }=\frac{1}{m} \sum_{i=1}^{m} \max _{1 \leq j \leq n} s_{i j} \\
& \text { columnScore }=\frac{1}{n} \sum_{j=1}^{n} \max _{1 \leq i \leq m} s_{i j}
\end{aligned}
$$

Using these definitions, the five similarity methods for the computation of gene similarity between two genes $g_{1}$ and $g_{2}$ are defined as follows:

$$
\begin{aligned}
& \operatorname{Sim}_{\text {Max }}\left(g_{1}, g_{2}\right)=\max _{1 \leq i \leq m, 1 \leq j \leq n} s_{i j} \\
& \operatorname{Sim}_{\text {Mean }}\left(g_{1}, g_{2}\right)=\frac{1}{m \times n} \sum_{i=1}^{m} \sum_{j=1}^{n} s_{i j} \\
& \operatorname{Sim}_{\text {funSimMax }}\left(g_{1}, g_{2}\right)=\max \{\text { rowScore, columnScore }\} \\
& \operatorname{Sim}_{\text {funSimAvg }}\left(g_{1}, g_{2}\right)=0.5 *(\text { rowScore }+ \text { columnScore }) \\
& \operatorname{Sim}_{\text {BMA }}\left(g_{1}, g_{2}\right)=\frac{\sum_{i=1}^{m} \max _{1 \leq j \leq n} s_{i j}+\sum_{j=1}^{n} \max _{1 \leq i \leq m} s_{i j}}{m+n}
\end{aligned}
$$

For a set of genes $G\left(g_{1}, g_{2}, \ldots, g_{n}\right)$ of size $n$, the similarity matrix for these genes is defined as $\mathrm{Sim}=$ $\left[\operatorname{Sim}_{i j}\right]_{n \times n}$, where $\operatorname{Sim}_{i j}$ is the similarity between gene $g_{1}$ and $g_{j}$ derived by any of the five methods defined above.

In DOSim, there are a total of fifty optional semantic similarity measures for genes, which are combinations of the ten semantic similarity measures for term pairs and the five similarity methods mentioned above.

\section{Other utilities}

\section{Conducting DO enrichment analysis}

In DOSim, DO-based enrichment analysis is implemented to explore the disease feature of an independent gene set, for example, a differentially expressed gene set from a microarray analysis. Significance of the enrichment analysis is assessed by the hypergeometric test and the $p$-value is adjusted by false discovery rate (FDR). For a certain DO term $t$ which meets the requirement (see below), if $M$ genes are the number of annotated genes in the human genome and $x$ genes are the number of annotated genes in the gene set for this term, then to calculate whether the gene set is enriched in DO term the following formula is used:

$$
p-\text { value }=1-\sum_{0 \leq i \leq x} \frac{C_{M}^{i} \times C_{N-M}^{k-i}}{C_{N}^{k}}
$$

where, $N$ is the total number of human genes in the genome, $k$ is the size of the gene set of interest, and $C_{N}^{k}$ is the number of combinations of the $N$ genes taken $k$ at a time and is equal to $\frac{N !}{k ! \times(N-k) !}$.

Compared with FunDO, which uses a small set of DO terms (DOLite) [13], DOSim selects the DO terms satisfy two criteria for enrichment analysis, aiming at exploring more biological result. The first criterion is that the term should be annotated by at least $n$ genes, and the second is that the term should be beneath a depth $m$ in the DAG of DO, where $n$ and $m$ can be set by users when running the $\mathrm{DO}$ enrichment analysis.

In the DOSim package, the DOEnrichment function carries out the DO enrichment analysis; the input is a list of Entrez gene IDs. The filter and layer parameters are the two criteria mentioned above that can be used to control the terms to be analysed; so that the term is annotated by at least 'filter size' genes and it is beneath the 'layer' depth in the DAG of DO.

\section{Detecting and annotating DO-directed gene modules}

A gene module is a group of highly correlated genes. In DOSim, gene modules can be detected as follows: after the gene similarity matrix for a gene set is constructed, a hierarchical clustering is performed using the standard $\mathrm{R}$ function hclust and one of three branch cutting methods is applied (one constant-height cutting and two dynamic branch cutting methods are embed in our package) [24].

The DOSim package incorporates multilayered enrichment analysis (GO and KEGG annotation) to explore the biological meaning of the detected gene modules. The GO annotations are conducted using GOSim [25] and the KEGG annotations are generated using 
SubpathwayMiner [26]. The input for GO and KEGG annotations is a list of Entrez gene IDs, the mechanism implied in each annotation database is the hypergeometric test, and the outputs for each annotation database are the enriched terms with $p$-values.

\section{Describing and visualizing DO structures and terms}

DO is a collection of terminologies associated with human diseases and the terms in DO are organised in a DAG (Figure 1). DOSim also provides useful utilities to easily visualise the DO structure; thus users need not turn to other tools (e.g., OBO-Edit). Specifically, the hierarchical structures of DO terms can be represented as a graphNEL object and the getDOGraph function in DOSim can be used to fetch the DO graph with specified DO terms at its leaves. For a certain DO term, DOSim provides a series of functions to extract related terms (e.g., father and child terms.).

\section{Results}

\section{The effect of different measures on the computation of} gene similarity

The different similarity measures for both the terms and the genes have their advantages when applied to biomedical ontologies [14]. An important question that we addressed was, do different similarity measures for the same gene pairs produce very different results? We used all the fifty similarity measures implemented in DOSim to calculate the similarities between the 4045 genes that have DO annotations. A Pearson correlation coefficient (PCC) analysis between the gene similarities calculated using the different similarity measures was then carried out to quantify the influence of the similarity measures. The resultant PCC frequency distribution (Figure 2) showed that the gene similarities calculated by the

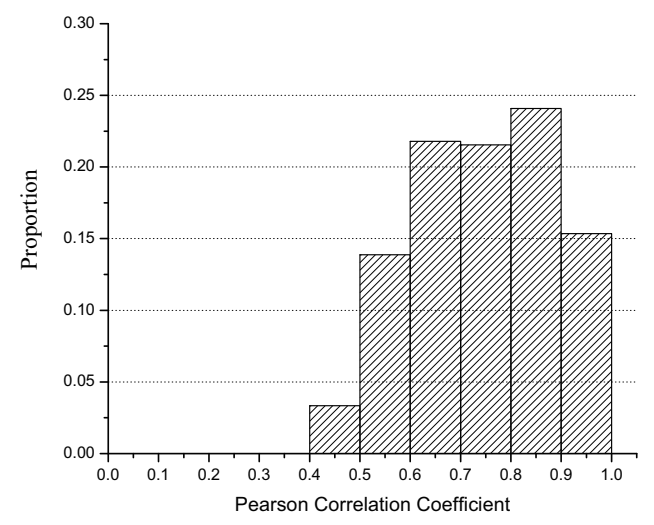

Figure 2 Distribution of the Pearson correlation coefficient of gene similarity scores between parameter combinations. different similarity measures were closely correlated, indicating that the different similarity measures do not much significantly influence the computation of gene similarity.

\section{Application on disease similarity}

We investigated the relationships between different kinds of cancers using disease similarities derived from DOSim. First, 128 cancer disease DO terms were obtained by using "cancer" as the key word to search all DO term names (exclude the DO term, "DOID:162, cancer"). Then, we used the getTermSim function to get the pairwise similarities using Wang measure (This is an example here. Users can choose any of the other measures in their applications).

Figure 3 is the average linkage hierarchical clustering of the 128 different cancer terms based on the similarities computed by the Wang measure. To assign significance to these associations, we randomly selected 128 diseases from all the diseases covered by DO terms and calculated the similarities among them. This process was repeated 100 times to generate a background distribution. The background distribution value at the 99th percentile was $0.43(p$-value $=0.01)$. Only those disease correlations that passed the $p$-value threshold of 0.01 were selected. Using this criterion we found 800 significant disease-disease similarity relationships. We defined a "module" as a subbranch in the hierarchical clustering which had at least three diseases and under a height of 0.57 (inverse of similarity). This resulted in 16 modules with sizes ranging from 3 to 22. Generally, many of the expected disease associations that pooled together in one sub-branch were those that we expected; for example, the thyroid-related cancers, well-differentiated thyroid cancer (DOID:3971), localised parathyroid cancer (DOID:1544), metastatic parathyroid cancer (DOID:7149) and recurrent parathyroid cancer (DOID:7150) were all in one module. Many novel and hitherto unknown significant correlations such as the similarity between hematologic cancer (DOID:2531) and spleen cancer (DOID:672) which had a similarity of 0.785 were discovered. The spleen is part of the lymphatic system which can filter the blood and help the body fight infections. Lymphoma is a type of hematologic cancer that develops in the lymphatic system. Malignant lymphoma can occur in various organs, including the spleen [27] and among the causes of isolated splenomegaly, lymphoid malignancies account for a relevant, yet probably underestimated, number of cases [28]. Taking the correlation between hematologic cancer and spleen as an example, such relationships can be easily explored by DOSim.

We also created a network representation to display all the 800 significant disease correlations by using the Cytoscape software package [29] (Figure 4). In the 


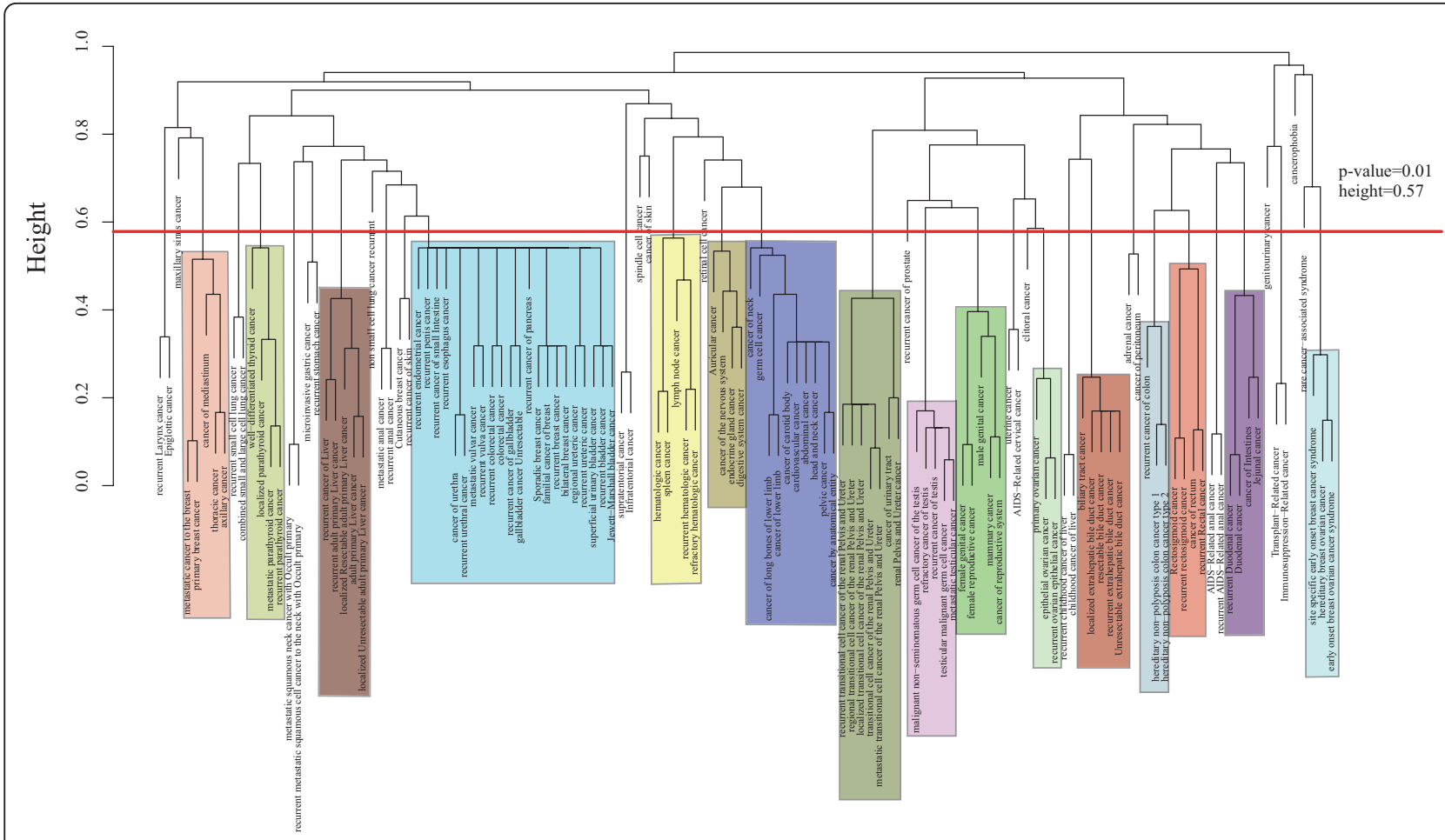

Figure 3 Hierarchical clustering of $\mathbf{1 2 8}$ cancer terms. The distance between two diseases is defined to be 1- the Wang's similarity of the two diseases. The tree was constructed using the average method of hierarchical clustering. The red line corresponds to a $p$-value of 0.01 . Disease correlations below this line are considered significant. The different colours represent the various categories of significant disease correlations.

network, the nodes were diseases, and the thickness of the edges between two diseases represented their strength of correlation. The network revealed strong correlations between different modules (defined in hierarchical clustering), which helped us to pick additional significant disease associations that were missing in the hierarchical clustering. For example, germ cell cancer (DOID:2994), a member of the module labelled in blue with size 10, correlated with almost every member of the largest module of size 22. This network application demonstrates that, although cancer diseases show modular characteristics, they are also highly correlated with each other. A detailed pairwise similarity matrix between the 128 cancer terms and a list of significant cancer pairs are provided in Additional file 1.

We also constructed the DO graph of these 128 cancers as leaves (Additional file 2), which finally contained 398 disease DO terms. We found that, as expected, diseases in the same module represented hierarchical structure in the DO graph as illustrated in the Figure S1. For example, the module marked brown contained 7 diseases, of which "cancer of urinary tract" (DOID:3996) is the ancestral node of the other 6 diseases. However, the observed correlation between "germ cell cancer" (DOID:2994) and the largest module which has a size of
22 (Figure 4) doesn't show any direct link in the DO graph. Again, the network representation in Figure 4 provided additional insights to our analysis.

\section{Application on gene similarity}

Here, by discussing the disease risk of obesity, we demonstrated another application of DOSim (using functions of calculating similarity between genes and DO-directed gene modules detection and annotation). Previous studies showed that obesity increased the risk of various diseases, such as type 2 diabetes, heart disease and certain types of cancer [30]. In this example, we used obesity related genes (651 genes) that were downloaded from the Phenopedia database[31]. Of the 651 genes, 361 had DO annotations. The similarities between these 361 genes were calculated using the BMA method on the Resnik measure (This is just one example. Users can choose to use any of the others in their applications). A gene similarity matrix $S=\left[s_{i j}\right]_{361} \times 361$ was constructed where $s_{i j}$ is the similarity between $i$ th gene and $j$ th gene in the gene set. After that an average linkage hierarchical clustering was performed and then a dynamic tree cutting method was applied (minimal module size is larger than 10) [24]. Finally, 10 different gene modules were obtained (Figure 5, Table 1). 


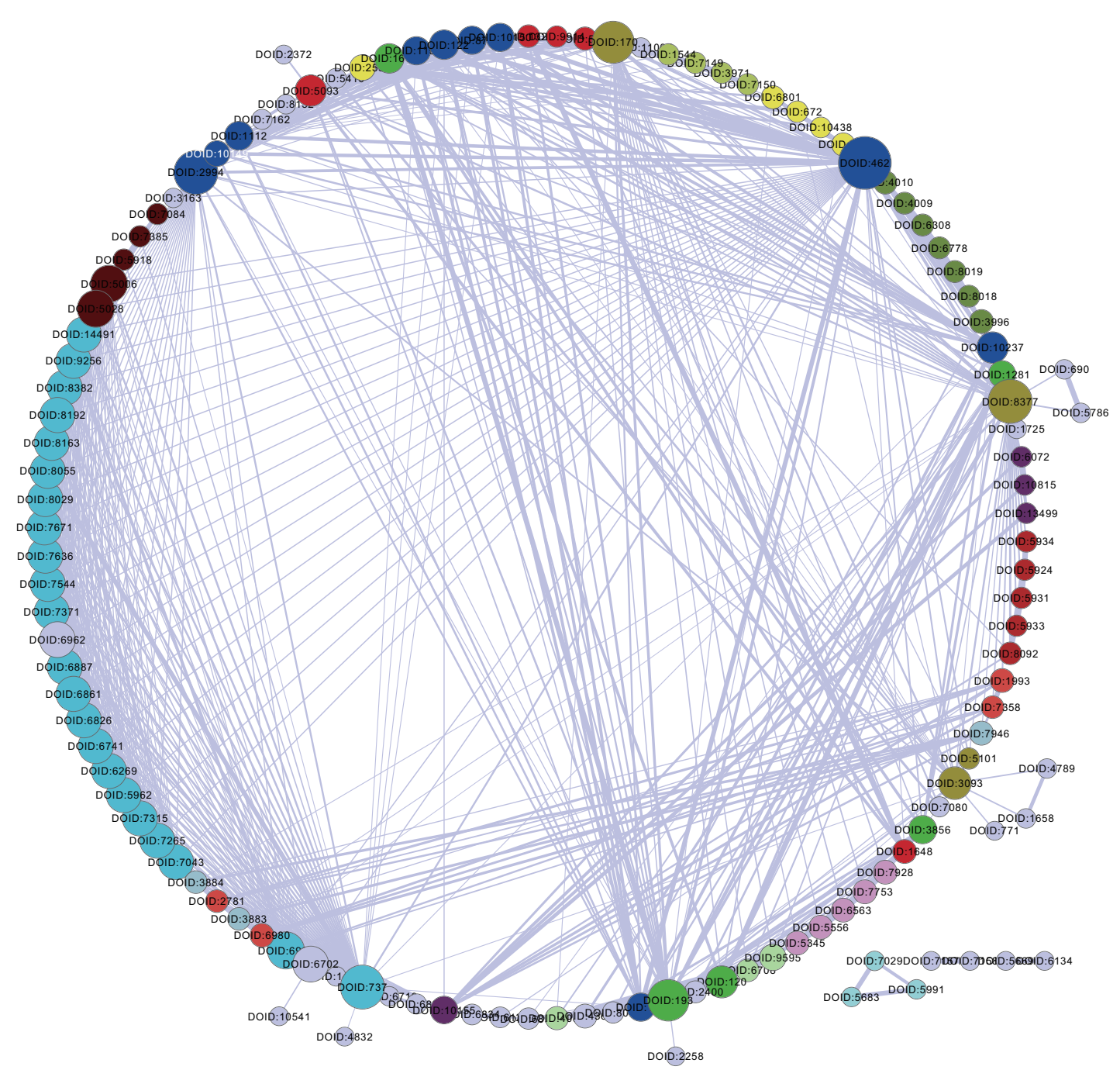

Figure 4 The network of all the 128 cancer terms. The colours correspond to the significant disease correlation categories in Figure 3 . The nodes coloured in grey are not grouped in Figure 3. The thickness of the edges between two diseases represents the strength of their correlation.

When the complete GO and KEGG annotations of these ten different gene modules were analysed (Additional file 3), we found different enriched biology functions and pathways for each module, indicating the complex pathogenesis of obesity. For example, the KEGG annotations of one of the clusters (M4) (Table 1) indicated that obesity is a factor that may lead to various cancers (e.g., colorectal cancer and endometrial cancer) and that obesity may also have a relationship with many signalling pathways (e.g., ErbB signalling pathway and Jak-STAT signalling pathway). However, the KEGG annotations of another cluster (M2) suggested that obesity may either affect the metabolism of many molecules or that the dysfunctional metabolism of these molecules may lead to the obesity (e.g., pyruvate metabolism and galactose metabolism). Similarly, the GO annotations of cluster M1 implied that obesity has a relationship with the biology process of cholesterol, lipoprotein and triglyceride (e.g., cholesterol homeostasis, reverse cholesterol transport, high-density lipoprotein particle remodelling and triglyceride catabolic process), while the GO annotations of cluster M3 suggested that obesity may be associated with eating habits (e.g., feeding behavior and drinking behavior). Both the GO and KEGG annotations of cluster M8 indicated that obesity is related to coagulation (blood coagulation in GO; complement and coagulation cascades in KEGG). These multilayered annotations successfully demonstrated the complex pathogenesis of obesity and suggested that the genes in the 


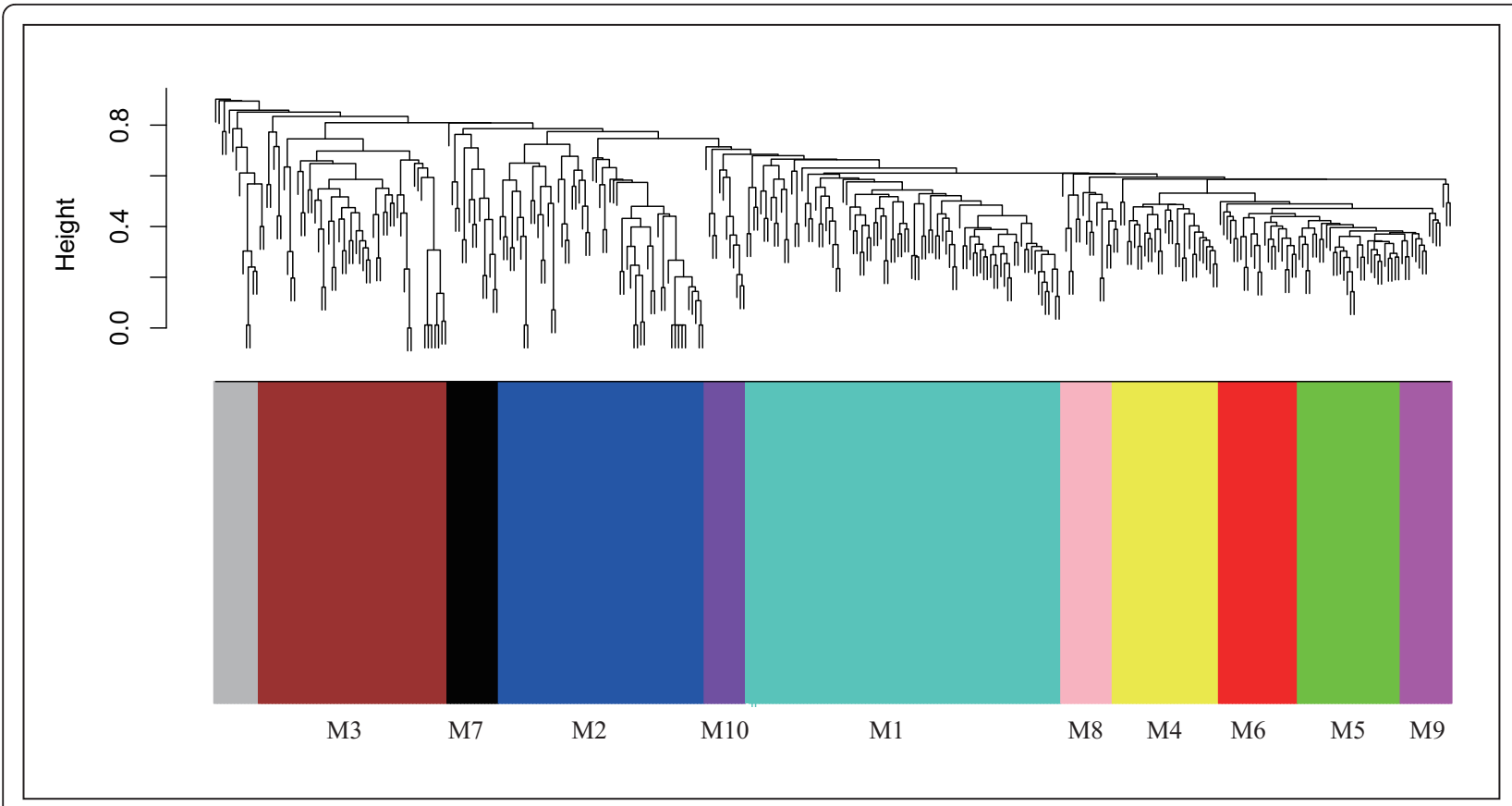

Figure 5 Hierarchical clustering result of the obesity related genes. The grey bar indicates the genes that could not be grouped into a certain module.

Table 1 Gene modules of the obesity related genes

\begin{tabular}{|c|c|c|c|c|c|c|}
\hline Cluster & Size & $\begin{array}{l}\text { Average } \\
\text { similarity }\end{array}$ & $p$-value $\#$ & FDR $^{*}$ & Representative GO annotation $^{\S}$ & Representative KEGG annotation $^{\S}$ \\
\hline M1 & 92 & 0.43 & $<1.0 \mathrm{E}-05$ & $<1.0 \mathrm{E}-04$ & $\begin{array}{l}\text { cholesterol homeostasis; high-density } \\
\text { lipoprotein particle remodelling; triglyceride } \\
\text { catabolic process }\end{array}$ & $\begin{array}{l}\text { Insulin signaling pathway; Type II } \\
\text { diabetes mellitus }\end{array}$ \\
\hline $\mathrm{M} 2$ & 60 & 0.30 & 0.25 & 0.28 & $N / A^{\$}$ & $\begin{array}{l}\text { Pyruvate metabolism; Galactose } \\
\text { metabolism; }\end{array}$ \\
\hline M3 & 55 & 0.30 & 0.29 & 0.29 & feeding behavior; photoreceptor cell maintenance & $\begin{array}{l}\text { Neuroactive ligand-receptor } \\
\text { interaction; Circadian rhythm - } \\
\text { mammal; }\end{array}$ \\
\hline M4 & 31 & 0.50 & $<1.0 \mathrm{E}-05$ & $<1.0 \mathrm{E}-04$ & $\begin{array}{l}\text { response to estrogen stimulus; response to } \\
\text { cytokine stimulus; cell aging }\end{array}$ & $\begin{array}{l}\text { Pathways in cancer; Colorectal } \\
\text { cancer; Endometrial cancer; }\end{array}$ \\
\hline M5 & 30 & 0.62 & $<1.0 \mathrm{E}-05$ & $<1.0 \mathrm{E}-04$ & $\begin{array}{l}\text { response to lipopolysaccharide; response } \\
\text { to glucocorticoid stimulus }\end{array}$ & $\begin{array}{l}\text { Cytokine-cytokine receptor } \\
\text { interaction; Toll-like receptor } \\
\text { signaling pathway; }\end{array}$ \\
\hline M6 & 23 & 0.55 & $<1.0 \mathrm{E}-05$ & $<1.0 \mathrm{E}-04$ & $\begin{array}{l}\text { positive regulation of phosphoinositide } 3 \text {-kinase } \\
\text { cascade; positive regulation of cholesterol } \\
\text { esterification }\end{array}$ & $\begin{array}{l}\text { Renin-angiotensin system; Prostate } \\
\text { cancer }\end{array}$ \\
\hline M7 & 15 & 0.34 & 0.12 & 0.16 & $\mathrm{~N} / \mathrm{A}$ & Insulin signaling pathway \\
\hline M8 & 15 & 0.43 & $6.0 \mathrm{E}-04$ & $6.0 \mathrm{E}-03$ & $\begin{array}{l}\text { blood coagulation; STAT protein nuclear } \\
\text { translocation }\end{array}$ & $\begin{array}{l}\text { Complement and coagulation } \\
\text { cascades; Regulation of actin } \\
\text { cytoskeleton }\end{array}$ \\
\hline M9 & 15 & 0.53 & $<1.0 \mathrm{E}-05$ & $<1.0 \mathrm{E}-04$ & $\begin{array}{l}\text { response to interleukin-1; response } \\
\text { to glucocorticoid stimulus }\end{array}$ & $\begin{array}{l}\text { Hematopoietic cell lineage; Cytokine- } \\
\text { cytokine receptor interaction }\end{array}$ \\
\hline M10 & 12 & 0.40 & 1.5E-02 & 2.2E-02 & N/A & N/A \\
\hline
\end{tabular}

\# the original $p$-value calculated by permutation

* FDR using Benjamini and Hochberg multiple testing correlations

$\S$ Refer to Additional file 3 for complete GO and KEGG annotations.

$\$ N / A$ indicates that there are no enriched GO or KEGG annotation for this module. 
different gene modules would be potential drug targets for the corresponding diseases caused by obesity.

\section{Discussion}

The DOSim package offers an easy and straight forward way to study disease similarity and gene similarity simultaneously in the DO. Additionally, other utilities implemented in the DOSim, such as function of gene module detection and gene module multilayered annotation, make better application of the DO and facilitate researchers. The presented two case studies highlight the usefulness of the DOSim in a real life scenario. We also provided the Additional file 4 which contains all the necessary $\mathrm{R}$ scripts to generate the above two case studies.

\section{Conclusions}

The DOSim package advances the use of DO by integrating information theoretic similarity concepts for diseases and deriving disease similarity measures for genes in the powerful $\mathrm{R}$ system. Compared with the few existing bioinformatics tools for DO, e.g., FunDO, which explores disease information implied in the gene set by enrichment analysis, DOSim focuses on the computation of disease-disease and gene-gene similarities. Other utilities, such as function for gene module detection and gene module multilayered annotation, should help promote a better understanding of the complex pathogenesis of some disease risk phenotypes and the heterogeneity of some diseases. DOSim is available on the Comprehensive R Archive Network (CRAN) project or through http:// bioinfo.hrbmu.edu.cn/dosim.

\author{
Availability and requirements \\ Project name: DOSim \\ Project home page: http://bioinfo.hrbmu.edu.cn/ \\ dosim \\ Operating system(s): platform independent \\ Programming language: $\mathrm{R}$ \\ Other requirements: none \\ License: GPL
}

\section{Additional material}

Additional file 1: Pairwise similarity matrix between 128 cancer terms and a list of significant cancer pairs. Similarities for these 128 cancers were computed by getTermSim function using the Wang measure. The threshold of similarity 0.43 was selected by permutation and the corresponding $p$-value was 0.01 . The excel file contains three separate sheets named 'readme', 'similarity matrix' and 'significant disease pairs'. They contain the following information: Readme: Brief introduction to the file. Similarity matrix: Stores all the 180 cancers' pairwise similarities. Data coloured red are those with a similarity larger than 0.43 , corresponding to $p$-value 0.01 . Significant disease pairs: Represents the significant disease pairs at a significant $p$-value of 0.01 fetched from the 'similarity matrix'.
Additional file 2: The DO graph of the 128 cancer DO terms. The DO graph of the 128 cancer DO terms was generated by "getDOGraph" function in the DOSim package. The 128 terms functioned as leaves, resulting in 378 terms in total. The 128 starting terms are represented as circles with different colours according to the modules they belong to. The additional 270 terms are represented as grey squares. Two modules coloured in brown and green are expanded as examples amd compared with the results in the Figure 3. Additionally, term DOID:2994 (germ cell cancer) is also expanded as an example and compared with the results in the Figure 4.

Additional file 3: Detailed annotation for ten obesity related gene modules Ten modules of obesity genes were obtained by

'detectModule' function with minimal module size larger than 10 and using the 'tree' method. The module annotation was carried out by the R script in the Addtional file 4 (R_Code.R'). All GO and KEGG terms assigned to each module are at a significant level of FDR $<=0.01$.

Additional file 4: $R$ and Perl scripts used to generate the results in the two case studies This zip file contains the 10 files, which were used to generate the results in the two case studies. Two files, the "R_Code.R" and the "get_significant_of_each_module.pl" are the main scripts that were used. A detailed description of all 10 files is available in the "Readme.txt" file.

\section{Acknowledgements and Funding}

This work is supported in part by the National Natural Science Foundation of China (Grant Nos. 30871394, 61073136 and 91029717), the Science Foundation of Heilongjiang Province (Grant Nos. ZD200816-01, JC200711, 2005-39, 1155H012, 11551232 and YJS(X2007-0195HL).

\section{Authors' contributions}

$J L, B G, C W, F Z, S R$ and $X L$ conceived the project and wrote the paper. $J L$, $X C, C L$ and $T L$ designed the software and performed the analyses. JL and $B G$ designed the code and implemented the software. All authors read and approved the final manuscript.

\section{Competing interests}

The authors declare that they have no competing interests.

Received: 16 January 2011 Accepted: 29 June 2011

Published: 29 June 2011

\section{References}

1. Kalaria R: Similarities between Alzheimer's disease and vascular dementia. J Neurol Sci 2002, 203-204:29-34.

2. Hu G, Agarwal P: Human disease-drug network based on genomic expression profiles. PLoS One 2009, 4(8):e6536.

3. Wang F, Syeda-Mahmood T, Beymer D: Finding Disease Similarity by Combining ECG with Heart Auscultation Sound. Computers in Cardiology 2007, 261-264

4. Goh Kl, Cusick ME, Valle D, Childs B, Vidal M, Barabasi AL: The human disease network. Proc Natl Acad Sci USA 2007, 104(21):8685-8690.

5. McKusick VA: Mendelian Inheritance in Man and its online version, OMIM. Am J Hum Genet 2007, 80(4):588-604.

6. Becker KG, Barnes KC, Bright TJ, Wang SA: The genetic association database. Nat Genet 2004, 36(5):431-432.

7. Liu YI, Wise PH, Butte AJ: The "etiome": identification and clustering of human disease etiological factors. BMC Bioinformatics 2009, 10(Suppl 2): S14.

8. Fowler J, Kouramajian V, Maram S, Devadhar V: Automated MeSH indexing of the World-Wide Web. Proc Annu Symp Comput Appl Med Care 1995, 893-897.

9. Zhang SH, Wu C, Li X, Chen X, Jiang W, Gong BS, Li J, Yan YQ: From phenotype to gene: detecting disease-specific gene functional modules via a text-based human disease phenotype network construction. FEBS Lett 2010, 584(16):3635-3643.

10. Harris MA, Clark J, Ireland A, Lomax J, Ashburner M, Foulger R, Eilbeck K, Lewis S, Marshall B, Mungall C, et al: The Gene Ontology (GO) database 
and informatics resource. Nucleic Acids Res 2004, 32(Database issue): D258-261.

11. Warren A, Kibbe J, Wolf W, Smith M, Zhu L, Lin S, Chisholm R: Disease Ontology. 2006.

12. Osborne J, Flatow J, Holko M, Lin S, Kibbe W, Zhu L, Danila M, Feng G, Chisholm R: Annotating the human genome with Disease Ontology. BMC Genomics 2009, 10(Suppl 1):S6.

13. Du P, Feng G, Flatow J, Song J, Holko M, Kibbe WA, Lin SM: From disease ontology to disease-ontology lite: statistical methods to adapt a general-purpose ontology for the test of gene-ontology associations. Bioinformatics 2009, 25(12):i63-68.

14. Pesquita C, Faria D, Falcão AO, Lord P, Couto FM: Semantic Similarity in Biomedical Ontologies. PLoS Comput Biol 2009, 5(7):e1000443.

15. Resnik P: Using Information Content to Evaluate Semantic Similarity in a Taxonomy. Proceedings of the 14th International Joint Conference on Artificial Intelligence, Montreal 1995, 1:448-453.

16. Lin D: An Information-Theoretic Definition of Similarity. ICML '98: Proceedings of the Fifteenth International Conference on Machine Learning 1998, 296-304

17. Jiang J, Conrath D: Semantic similarity based on corpus statistics and lexical taxonomy. Proceedings of the International Conference on Research in Computational Linguistics, Taiwan 1998.

18. A. Schlicker FD: A new measure for functional similarity of gene products based on Gene Ontology. BMC Bioinformatics 2006.

19. C. Pesquita DF: Evaluating GO-based Semantic Similarity Measures. In: Proc 10th Annual Bio-Ontologies Meeting 2007, 37-40.

20. A. Feltus B, Li JW: Effectively Integrating Information Content and Structural Relationship to Improve the GO-based Similarity Measure Between Proteins. BMC Bioinformatics 2009.

21. James Z, Wang ZD: A new method to measure the semantic similarity of GO terms. Bioinformatics 2007, 1274-1281.

22. Couto F, Silva M, Coutinho P: Semantic Similarity over the Gene Ontology: Family Correlation and Selecting Disjunctive Ancestors. Conference in Information and Knowledge Management 2005.

23. Lord PW, Stevens RD, Brass A, Goble CA: Investigating semantic similarity measures across the Gene Ontology: the relationship between sequence and annotation. Bioinformatics 2003, 19(10):1275-1283.

24. Langfelder $\mathrm{P}$, Zhang $B$, Horvath $\mathrm{S}$ : Defining clusters from a hierarchical cluster tree: the Dynamic Tree Cut package for R. Bioinformatics 2008, 24(5):719-720.

25. Frohlich H, Speer N, Poustka A, BeiSZbarth T: GOSim: an R-package for computation of information theoretic GO similarities between terms and gene products. BMC Bioinformatics 2007, 8(1):166.

26. Li C, Li X, Miao Y, Wang Q, Jiang W, Xu C, Li J, Han J, Zhang F, Gong B, et al: SubpathwayMiner: a software package for flexible identification of pathways. Nucleic Acids Res 2009, 37(19):e131.

27. Tokoro Y: Cytology of malignant lymphoma. Rinsho Byori 2010, 58(11):1113-1120.

28. Iannitto $E$, Tripodo C: How I diagnose and treat splenic lymphomas. Blood 2010, 117(9):2585-2595.

29. Cline MS, Smoot M, Cerami E, Kuchinsky A, Landys N, Workman C, Christmas R, Avila-Campilo I, Creech M, Gross B, et al: Integration of biological networks and gene expression data using Cytoscape. Nat Protoc 2007, 2(10):2366-2382.

30. Haslam DW, James WP: Obesity. Lancet 2005, 366(9492):1197-1209.

31. Yu W, Clyne M, Khoury MJ, Gwinn M: Phenopedia and Genopedia: disease-centered and gene-centered views of the evolving knowledge of human genetic associations. Bioinformatics 2009, 26(1):145-146.

doi:10.1186/1471-2105-12-266

Cite this article as: Li et al: DOSim: An R package for similarity between diseases based on Disease Ontology. BMC Bioinformatics 2011 12:266.

\section{Submit your next manuscript to BioMed Central and take full advantage of:}

- Convenient online submission

- Thorough peer review

- No space constraints or color figure charges

- Immediate publication on acceptance

- Inclusion in PubMed, CAS, Scopus and Google Scholar

- Research which is freely available for redistribution

Submit your manuscript at www.biomedcentral.com/submit
C Biomed Central 